\title{
Role of Resonance Energy Transfer in Light Harvesting of Zinc Oxide-Based Dye-Sensitized Solar Cells
}

\author{
Abhinandan Makhal, ${ }^{\dagger}$ Soumik Sarkar, ${ }^{\dagger}$ Tanujjal Bora, ${ }^{\dagger}$ Sunandan Baruah,${ }^{\ddagger}$ Joydeep Dutta, ${ }^{\ddagger}$ \\ A. K. Raychaudhuri, ${ }^{\dagger}$ and Samir Kumar Pal ${ }^{*}$, \\ Unit for Nano Science \& Technology, S. N. Bose National Centre for Basic Sciences, Block JD, Sector III, \\ Salt Lake, Kolkata 700 098, India, and Centre of Excellence in Nanotechnology, School of Engineering and \\ Technology, Asian Institute of Technology, Klong Luang, Pathumthani 12120, Thailand
}

Received: March 6, 2010; Revised Manuscript Received: April 28, 2010

In this contribution we have studied the dynamics of light harvesting of $\mathrm{ZnO}$ nanoparticles (NPs) to a surface adsorbed sensitizing dye (SD) N719. By using the picosecond resolved Förster resonance energy transfer (FRET) technique we have explored that the excited $\mathrm{ZnO}$ NPs resonantly transfer visible optical radiation to the SD N719. The consequence of the energy transfer on the performance of the overall efficiency of a model $\mathrm{ZnO}$ NP-based dye-sensitized solar cell (DSSC) has also been explored. We have demonstrated that the overall efficiency of a ZnO NP-based solar cell significantly depends on the presence of high-energy photons in the solar radiation. In a control experiment on a model $\mathrm{TiO}_{2}$ NP-based solar cell it has been demonstrated that the presence of high-energy photon has a minimal effect on the performance of the cell as the $\mathrm{TiO}_{2} \mathrm{NPs}_{\text {are }}$ incapable of harvesting high-energy photons from solar radiation. The possibility of the back electron transfer from the excited NPs to the SD has also been investigated by studying the NPs in the presence of an ideal electron accepting organic molecule, benzoquinone (BQ). The time constants and nonradiative rate constant obtained for the $\mathrm{ZnO} / \mathrm{N} 719$ system are found to be different from those of the $\mathrm{ZnO} / \mathrm{BQ}$ system, which rules out the possibility of back electron transfer from ZnO NPs to SD N719. Moreover, the observed FRET dynamics in the light harvesting process of the nanocrystalites may be efficient in the further use of the nanoparticles in the development of new photodevices.

\section{Introduction}

The promising dye-sensitized solar cell (DSSC) is a relatively new class of low-cost solar cells suitable for renewable electricity generation. ${ }^{1,2}$ It is based on solar light harvesting of a sensitizing dye (SD) attached to a wide band gap semiconductor. The process of conversion of solar energy to electrical energy in a DSSC involves SD adsorbed on the surface of wide band gap n-type metal oxide semiconductor nanoparticles (typically $\mathrm{TiO}_{2},{ }^{3} \mathrm{ZnO},{ }^{4,5} \mathrm{SnO}_{2},{ }^{6} \mathrm{Nb}_{2} \mathrm{O}_{5},{ }^{7}$ etc). During operation, photons intercepted by the SD molecules create electron-hole pairs that are rapidly separated at the nanoparticle surface, with electrons injected into the semiconductor nanoparticles and holes leaving the opposite side of the device by means of redox species (usually $\mathrm{I}^{-} / \mathrm{I}_{3}{ }^{-}$) in a electrolyte in liquid ${ }^{8}$ or solid state. ${ }^{5}$ State of the art $\mathrm{TiO}_{2}$ nanoparticle based DSSC, which primarily absorbs light in the region of 350-700 nm, offer power conversion efficiency of only $11 \% .{ }^{9}$ It has been recognized that the key improvement of the efficiency of DSSC is to increase their spectral absorption range. In a recent study it has been estimated that a DSSC with power conversion efficiency of $15 \%$ using a I $\mathrm{I}^{-} / \mathrm{I}_{3}{ }^{-}$redox couple must absorb $\sim 80 \%$ of the solar spectrum from 350 to $900 \mathrm{~nm} .{ }^{10}$

Light absorption in DSSCs is determined by the molar extinction coefficient of the SD, the surface coverage of the dye (dye molecules $\mathrm{nm}^{-2}$ ), and the total surface area of the oxide film. ${ }^{11}$ The SD has traditionally been made from rutheniumbased complexes (for example, N719 and Z907), ${ }^{9}$ that have

\footnotetext{
* To whom correspondence should be addressed.E-mail: skpal@ bose.res.in.

$\dagger$ S. N. Bose National Centre for Basic Sciences.

* Asian Institute of Technology.
}

fairly broad absorption spectra $(\Delta \lambda \approx 350 \mathrm{~nm})$ but low molar extinction coefficients $\left(5000-20000 \mathrm{M}^{-1} \mathrm{~cm}^{-1}\right)$. Organic dyes have recently been developed with substantially higher molar extinction coefficients $\left(50000-200000 \mathrm{M}^{-1} \mathrm{~cm}^{-1}\right)$ but have very narrow spectral bandwidths $(\Delta \lambda \approx 250 \mathrm{~nm}) .{ }^{13}$ As a general rule, dyes that absorb strongly do not typically exhibit broad absorption characteristics. ${ }^{14}$ In an attempt to enhance light absorption and broaden the spectral response, organic DSSC, , $^{15}$ cosensitization of the semiconductor nanoparticles (titania) by dyes with complementary absorption spectra, has recently been demonstrated. However, limitations in the number of available sites at the surface of the semiconductor nanoparticles for the cosensitizing dyes constrains the total light absorbed in the solar cells.

To improve the possibility of enhanced light absorption, the use of Förster resonance energy transfer (FRET) between covalently linked energy donor molecules to the SD attached on the semiconductor (titania) surface has been demonstrated in a recent publication. ${ }^{16}$ One of the studies was able to demonstrate high excitation transfer efficiency $(>89 \%)$ between attached dye molecules and an improvement in the device external quantum efficiency of 5-10\% between the 400 and $500 \mathrm{~nm}$ spectral range. However, the overall power conversion efficiency enhancement of the DSSC was low $(<9 \%)$, and was argued to arise due to an increase in the open circuit voltage rather than an increase in the short-circuit photocurrent density. More recently, it has been demonstrated that unattached, highly luminescent chromophores inside liquid electrolyte can absorb high-energy photons and efficiently transfer the energy to the anchored near-infrared SD leading to an increase in optical absorption efficiency. ${ }^{14}$ 
Although most of the reported works on DSSC are based on $\mathrm{TiO}_{2}$ porous thin films, various structures of $\mathrm{ZnO}$ are also being used for DSSC fabrication. ${ }^{5,17-19}$ The advantages of using $\mathrm{ZnO}$ over $\mathrm{TiO}_{2}$ are its direct band gap $(3.37 \mathrm{eV})$, higher exciton binding energy (60 meV) compared to $\mathrm{TiO}_{2}(4 \mathrm{meV}),{ }^{20,21}$ and higher electron mobility $\left(200 \mathrm{~cm}^{2} \mathrm{~V}^{-1} \mathrm{~s}^{-1}\right)$ over $\mathrm{TiO}_{2}\left(30 \mathrm{~cm}^{2}\right.$ $\left.\mathrm{V}^{-1} \mathrm{~s}^{-1}\right) .{ }^{22}$ However, the efficiency of the DSSC based on $\mathrm{ZnO}$ nanostructures is still very low $(5 \%) .{ }^{23}$ One of the possibilities to enhance the efficiency of ZnO-based DSSC is the enhancement of the light absorbing ability of the DSSC. It has to be noted that $\mathrm{ZnO}$ can absorb high-energy photons $(>3.37 \mathrm{eV})$ and offers photoluminescence (PL) in the visible region $(2.25 \mathrm{eV}) .^{24}$

Careful use of the defect mediated $\mathrm{ZnO}$ PL for the excitation of the surface adsorbed SD, in order to enhance the overall efficiency of the ZnO-based DSSC, is the motive of the present study. In this article we have explored the FRET dynamics from a $\mathrm{ZnO}$ semiconductor nanoparticle to a surface adsorbed N719 dye used as sensitizer. By using steady state picosecond resolved fluorescence spectroscopy we have demonstrated that PL from a $\mathrm{ZnO}$ nanoparticle can be used to excite the SD molecule for the enhancement of light absorption possibility. The study also reveals that physical migration of the photoinduced charge from the $\mathrm{ZnO}$ nanoparticles to the SD molecules is insignificantly small in the de-excitation of the semiconductor nanoparticles. The effect of the ZnO NPs mediated light harvesting process on the overall efficiency of a model DSSC has also been demonstrated.

\section{Materials and Methods}

Fabrication of Dye-Sensitized Solar Cell. $\mathrm{ZnO}$ nanoparticles were synthesized in a colloidal solution with ethanol $\mathrm{C}_{2} \mathrm{H}_{5} \mathrm{OH}$ (J.T. Baker) as the solvent. The coprecipitation technique has been reported in previous publications from the group. ${ }^{25-28}$ Briefly $40 \mathrm{~mL}$ of $2 \mathrm{mM}$ zinc acetate dihydrate $\left(\mathrm{CH}_{3} \mathrm{COO}\right)_{2} \mathrm{Zn} \cdot 2 \mathrm{H}_{2} \mathrm{O}$ (Merck) solution was heat treated at 70 ${ }^{\circ} \mathrm{C}$ for half an hour. Next $20 \mathrm{~mL}$ of a $4 \mathrm{mM}$ sodium hydroxide $\mathrm{NaOH}$ (Merck) solution in ethanol was then added and the admixture was hydrolyzed for $2 \mathrm{~h}$ at $60{ }^{\circ} \mathrm{C}$. The colloidal solution of $\mathrm{ZnO}$ NPs is used to perform all the spectroscopic studies. The DSSC photoelectrode was prepared by in situ synthesis of $\mathrm{ZnO}$ quantum dots on commercial fluorinated tin oxide (FTO) glass substrate (Asahi, Japan), using a simple hydrothermal process. ${ }^{29} \mathrm{ZnO}$ NPs were synthesized on to the substrate by dropping ethanolic solutions of $1 \mathrm{mM}$ zinc acetate dihydrate followed by annealing at $350{ }^{\circ} \mathrm{C}$ for $5 \mathrm{~h}$.

The $\mathrm{ZnO}$ nanoparticle photoelectrode was then soaked in a $0.5 \mathrm{mM}$ ethanolic solution of dye N719 (Solaronix, Switzerland) for $24 \mathrm{~h}$ for the adsorption of dye and rinsed properly with dry ethanol. A platinized FTO glass $8 \Omega$ /square (Asahi, Japan) was used as counter electrode. The platinum layer was deposited on the FTO surface by thermal decomposition of platinum chloride, $\left[\mathrm{H}_{2} \mathrm{PtCl}_{6} \cdot \mathrm{H}_{2} \mathrm{O}\right]$ (Fluka), at $385{ }^{\circ} \mathrm{C}$ for $15 \mathrm{~min}$. The counter electrode was then placed on top of the photoelectrode and a single layer of $50 \mu \mathrm{m}$ thick Surlyn 1720 (Dupont) was used as a spacer between the two electrodes. The DSSC was then sealed properly by using the DSSC assembly machine developed in our lab. The liquid electrolyte composed of the 0.5 M LiI, 0.05 $\mathrm{M} \mathrm{I}_{2}$, and 0.5 M 4-tert-butylpyridine (TBP) in acetonitrile (ACN) was then filled in the cell using capillary force through small holes drilled on the counter electrode. Finally the holes in the counter electrode were sealed to prevent the electrolyte from leaking. $I-V$ characteristic measurements of the DSSC were performed under AM1.5G sun irradiation $\left(100 \mathrm{~mW} / \mathrm{cm}^{2}\right)$, using a $150 \mathrm{~W}$ small beam simulator (Sciencetech, model SF150).

Characterization Methods. High resolution TEM experiments are performed in FEI (Technai S-twin) instrument at SINP, India. For optical experiments, the steady state absorption and emission are determined with a Shimadzu UV-2450 spectrophotometer and a Jobin Yvon Fluoromax-3 fluorimeter (pump power at $320 \mathrm{~nm}$ is $\sim 22 \mu \mathrm{W} / \mathrm{cm}^{2}$ ), respectively. Picosecond-resolved spectroscopic studies were done with a commercial time correlated single photon counting (TCSPC) setup from Edinburgh Instruments (instrument response function $(I R F)=60 \mathrm{ps}$; excitation at $375 \mathrm{~nm}$ ). The picosecond resolved decay curves are fitted by nonlinear least-squares method to the triexponential decay law as given by the expression $\sum_{i}{ }^{3}=1$ $A_{i} \exp \left(-t / \tau_{i}\right)$, where, $A_{i}$ is the weight percentage of the decay components with time constants of $\tau_{i}$. The average excited lifetime is calculated by the following relation: $\tau=$ $\sum_{i=1}^{3} A_{i} \tau_{i} / \sum_{i=1}^{3} A_{i}$.

We have studied the complexation between $\mathrm{ZnO} \mathrm{NP}$ with different concentrations of N719. The ratio of the fluorescence intensity of the ZnO NP in the absence $\left(I_{0}\right)$ and presence $(I)$ of the quencher N719 can be written in Stern-Volmer equation. ${ }^{30}$

$$
\frac{I_{0}}{I} \text { or } \frac{\tau_{0}}{\tau}=1+K_{\mathrm{SV}}[Q]
$$

where, $I_{0} / I$ is the relative steady state emission intensity, $\tau_{0} / \tau$ is the relative excited state lifetime, and $K_{\mathrm{SV}}$ is the Stern-Volmer constant.

To estimate fluorescence resonance energy transfer efficiency of the donor $(\mathrm{ZnO})$ and hence to determine distance of donor-acceptor pairs we have used the following methodology. ${ }^{30}$ The Förster distance $\left(R_{0}\right)$ is given by

$$
R_{0}=0.211 \times\left[\kappa^{2} n^{-4} Q_{\mathrm{D}}\right]^{1 / 6}
$$

where $\kappa^{2}$ is a factor describing the relative orientation in space of the transition dipoles of the donor and acceptor. For donor and acceptor that randomize by rotational diffusion prior to energy transfer, the magnitude of $\kappa^{2}$ is assumed to be $2 / 3$. The refractive index $(n)$ of the medium is assumed to be 1.4. $Q_{\mathrm{D}}$, the integrated quantum yield of the donor in the absence of acceptor, is measured to be $3.8 \times 10^{-3}$. $J$, the overlap integral, which expresses the degree of spectral overlap between the donor emission intensity (normalized to unit area) ${ }^{31}$ and the acceptor absorption, is given by

$$
J=\frac{\int_{0}^{\infty} F_{\mathrm{D}}(\lambda) \varepsilon_{\mathrm{A}}(\lambda) \lambda^{4} \mathrm{~d} \lambda}{\int_{0}^{\infty} F_{\mathrm{D}}(\lambda) \mathrm{d} \lambda}
$$

where $F_{\mathrm{D}}(\lambda)$ is the fluorescence intensity of the donor in the wavelength range of $\lambda$ to $\lambda+\mathrm{d} \lambda$ and is dimensionless. $\varepsilon_{\mathrm{A}}(\lambda)$ is the extinction coefficient (in $\mathrm{M}^{-1} \mathrm{~cm}^{-1}$ ) of the acceptor at $\lambda$. If $\lambda$ is in $\mathrm{nm}$, then $J$ is in units of $\mathrm{M}^{-1} \mathrm{~cm}^{-1} \mathrm{~nm}^{4}$. The estimated value of the overlap integral is $6.751 \times 10^{14} \mathrm{M}^{-1} \mathrm{~cm}^{-1} \mathrm{~nm}^{4}$.

Once the value of $R_{0}$ is known, the donor-acceptor distance ( $r$ ) can be easily calculated by using the formula

$$
r^{6}=\frac{\left[R_{0}{ }^{6}(1-E)\right]}{E}
$$



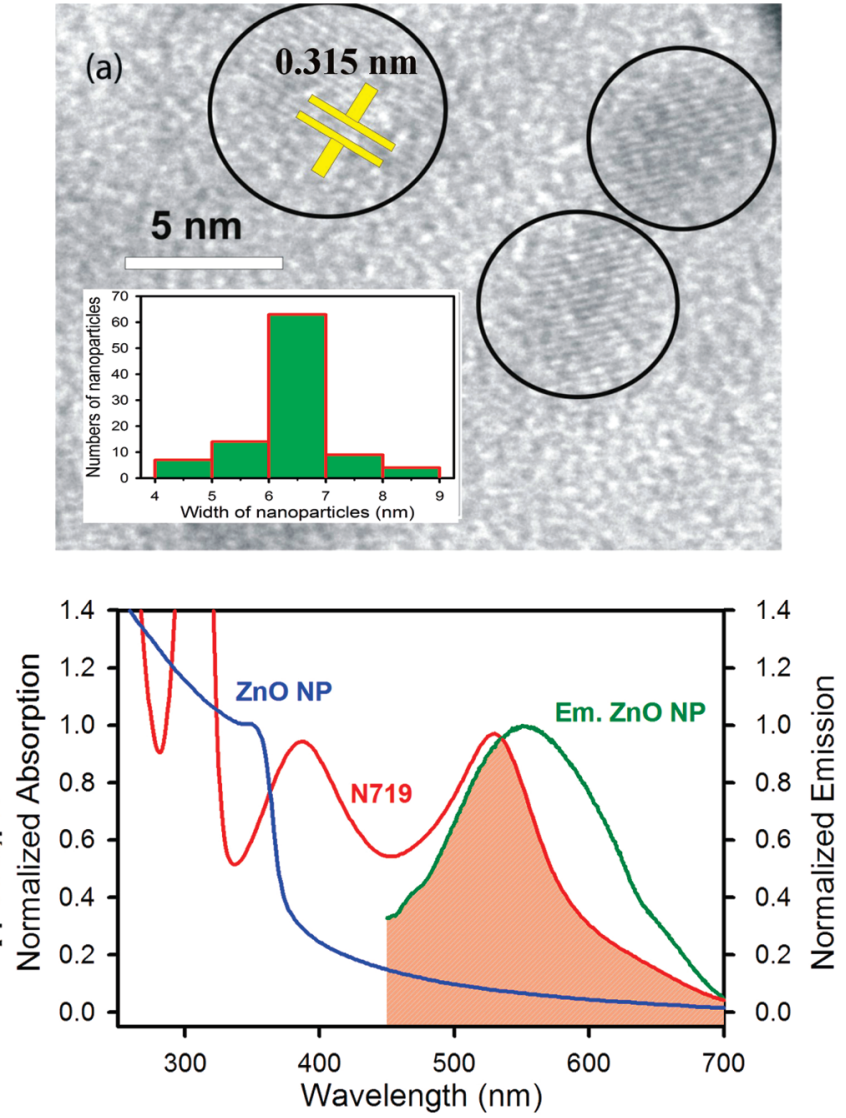

Figure 1. (a) High-resolution transmission electron micrograph (HRTEM) showing the $\mathrm{ZnO}$ nanoparticles with $\sim 3 \mathrm{~nm}$ radius. The inset shows the size distribution of the ZnO NP. (b) Steady state absorption spectra of ZnO NP, N719 and emission spectra of ZnO NP are shown. An overlapping zone between emission of ZnO NP and absorption of acceptor N719 is indicated as a red shaded zone.

Here $E$ is the efficiency of energy transfer. The transfer efficiency is measured by using the relative fluorescence lifetime of the donor, in the absence $\left(\tau_{\mathrm{D}}\right)$ and presence $\left(\tau_{\mathrm{DA}}\right)$ of the acceptor.

$$
E=1-\frac{\tau_{\mathrm{DA}}}{\tau_{\mathrm{D}}}
$$

We are also interested in obtaining the thickness of the surface layer emitting visible light by using a simple model. ${ }^{32}$ To obtain the below band gap $(550 \mathrm{~nm})$ and near band edge $(365 \mathrm{~nm})$ emission of the $\mathrm{ZnO}$ nanoparticles, we have excited the sample with $310 \mathrm{~nm}$ light. The luminescence peak intensity ratio of the near band edge (NBE) to below band gap (BBG) emission for spherical particles of radius $r$ and with a surface recombination layer of thickness $t$ is given by ${ }^{33}$

$$
\frac{I_{\mathrm{NBE}}}{I_{\mathrm{BBG}}}=C\left(\frac{r^{3}}{3 r t(r-t)+t^{3}}-1\right)
$$

The constant $C$, along with other quantities, contains the oscillator strengths which in turn depend on the particle morphology. To calculate $t$ from the above equation we have taken the magnitude of $C$ as 3.89 for small spherical particles ${ }^{33}$ with radius $r=3 \mathrm{~nm}$. For our system $\mathrm{NBE}(365 \mathrm{~nm}) / \mathrm{BBG}(550$ $\mathrm{nm})$ was found to be 0.853 . By inserting the values in eq 6 the
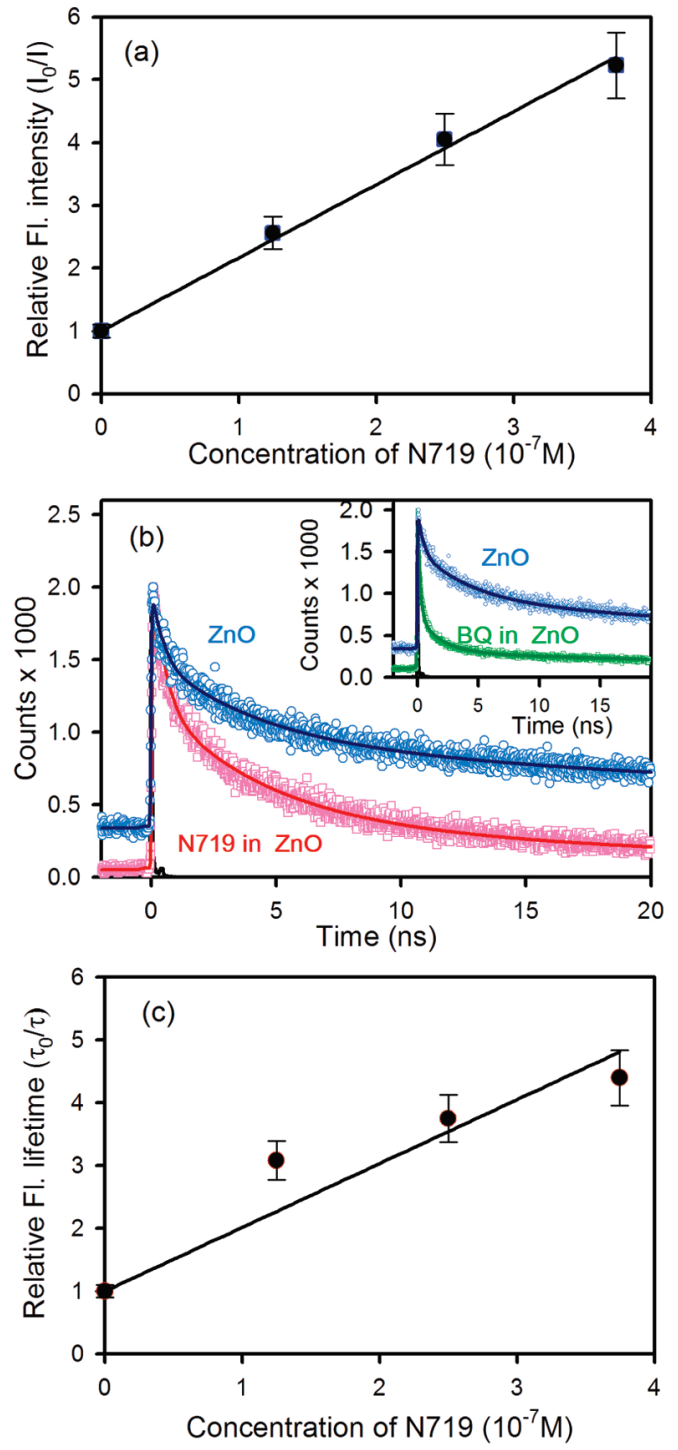

Figure 2. (a) Steady state emission intensity of $\mathrm{ZnO}$ nanoparticles (excitation at $375 \mathrm{~nm}$ ) in the presence of increasing concentration of N719 dye relative to that of $\mathrm{ZnO}$ emission without N719. (b) The picosecond resolved fluorescence transients of $\mathrm{ZnO} \mathrm{NP}$, in the absence (blue) and the presence of acceptor N719 (red) (excitation at $375 \mathrm{~nm}$ ) collected at $550 \mathrm{~nm}$. The inset shows the faster PL decay of $\mathrm{ZnO}-\mathrm{NP}$ in the presence of BQ. (c) Excited state lifetime of the PL emission of $\mathrm{ZnO}$ nanoparticles (excitation at $375 \mathrm{~nm}$ ) in the presence of increasing concentration of $\mathrm{N} 719$ dye relative to that of $\mathrm{ZnO}$ emission without N719 is shown.

thickness of the surface layer, $t$, is calculated. It represents an effective distance from the surface (effective diffusion length), within which the excited carriers recombine at the surface. The simple model above thus establishes that the visible emission centers are indeed located in a small region near the surface of the $\mathrm{ZnO}$ NP. The observed FRET dynamics, as we will see below, is also supporting evidence of the above fact.

\section{Results and Discussions}

The high-resolution transmission electron microscopic (HRTEM) image of the $\mathrm{ZnO}$ nanoparticles (NPs) is shown in Figure 1a. A predominance of nanoparticles with diameters of 6-7 $\mathrm{nm}$ were found in the synthesized colloids [inset of Figure 1a]. Figure $1 \mathrm{~b}$ shows the UV/vis absorption spectra of $\mathrm{ZnO} \mathrm{NP}$ and dye N719. The photoluminescence (PL) spectrum of the $\mathrm{ZnO}$ NPs upon excitation with $375 \mathrm{~nm}$ is also shown in the figure. 
TABLE 1: Picosecond Resolved Luminescence Transients of ZnO NPs in the Presence and Absence of N719 ${ }^{a}$

\begin{tabular}{|c|c|c|c|c|}
\hline sample & $\tau_{1}$ & $\tau_{2}$ & $\tau_{3}$ & $\tau_{\text {avg }}$ \\
\hline ZnO NP (bare) & $47.58 \mathrm{~ns}(41 \%)$ & $3.78 \mathrm{~ns}(23 \%)$ & $0.280 \mathrm{~ns}(36 \%)$ & $20.32 \mathrm{~ns}$ \\
\hline $\mathrm{ZnO} \mathrm{NP}+\mathrm{N} 719$ dye & 29.12 ns $(12 \%)$ & 3.86 ns $(38 \%)$ & 0.344 ns $(50 \%)$ & $5.25 \mathrm{~ns}$ \\
\hline $\mathrm{ZnO} \mathrm{NP}+\mathrm{BQ}$ & $30.20 \mathrm{~ns}(6 \%)$ & $1.93 \mathrm{~ns}(18 \%)$ & $0.187 \mathrm{~ns}(76 \%)$ & $2.20 \mathrm{~ns}$ \\
\hline
\end{tabular}

${ }^{a}$ The emission from $\mathrm{ZnO}$ nanoparticles (emission at $550 \mathrm{~nm}$ ) was detected with $375 \mathrm{~nm}$ excitation laser excitation. Numbers in the parentheses indicate relative weightage.

Significant spectral overlap of the ZnO PL spectrum with that of the absorption of the N719, which justifies the possibility of energy transfer from $\mathrm{ZnO}$ to the N719 SD molecules, is clearly evident from the figure. To investigate the complexation of $\mathrm{ZnO}$ NPs with the SD N719, we have studied the steady state PL of the ZnO NPs in the presence of various concentrations of N719 as shown in Figure 2a. We have also studied the PL transient at $550 \mathrm{~nm}$ (excitation $375 \mathrm{~nm}$ ) (Table 1). The transients in the absence and presence of various N719 concentrations are shown in Figure $2 b$. The PL quenching as evident from the steady state and time-resolved PL studies shows the affinity of the N719 dyes with the ZnO NPs. Detailed Stern-Volmer (S-V) analysis on the quenching of the $\mathrm{ZnO} \mathrm{PL}$ (as shown in Figure 2a,c) reveals the $\mathrm{S}-\mathrm{V}$ constants to be $K_{\mathrm{SV}}($ steady state $)=1.14 \times$ $10^{7} \mathrm{M}^{-1} \mathrm{~S}^{-1}$ and $K_{\mathrm{SV}}($ time-resolved $)=0.87 \times 10^{7} \mathrm{M}^{-1} \mathrm{~S}^{-1}$, respectively. The similarity of $K_{\mathrm{SV}}$ constants from steady state and time-resolved measurements indicates the $\mathrm{ZnO}$ PL quenching to be dynamic in nature. In other words, the possibility of formation of nonfluorescent $\mathrm{ZnO} \mathrm{SD}$ complex in the ground state is found to be negligibly small.

The dynamic nature of the PL quenching of ZnO NPs upon complexation with N719 is further justified from the FRET of the PL to the surface adsorbed SD N719. The overall FRET efficiency is found to be $74 \%$. In this case the spectral overlap integral $(J$, eq 3$)$ and the Förster distance $\left(R_{0}\right)$ are found to be $6.597 \times 10^{14}$ and $1.84 \mathrm{~nm}$, respectively. From FRET dynamics, it has to be noted that the effective distance $\left(r_{\mathrm{DA}}\right)$ between the donor ( $\mathrm{ZnO} \mathrm{NP}$ ) and the acceptor (N719) is $1.54 \mathrm{~nm}$, which is much lower than that of the average radii of the nanocrystals $(\sim 3 \mathrm{~nm})$. In a FRET study ${ }^{34}$ on cadmium selenide (CdSe) quantum dot (donor) and a surface adsorbed organic dye (ethidium bromide; acceptor), the donor-acceptor distance was found to be close to the radius of the quantum dot. The relatively shorter donor-acceptor distance compared to the radius of the $\mathrm{ZnO}$ nanocrystallites in the present study can be rationalized from the fact that the origin of the photoluminescence peaking at $550 \mathrm{~nm}$ essentially arises from the crystallites exposed in the surface of the ZnO NPs. It is important to note that the thickness of the surface layer $(t)$ obtained from calculation (using eq 6) is found to be $1.30 \mathrm{~nm}$, which is comparable to the effective distance between the donor and acceptor calculated from the FRET study. The observation is consistent with the fact that there is a surface region from where the defect related emission occurs.

To investigate the effect of light harvesting of high-energy photons by the host $\mathrm{ZnO}$ semiconductor in a model DSSC, we have studied the current-voltage $(I-V)$ characteristics as shown in Figure 3a. We measured $I-V$ characteristics of the solar cells in two different experimental conditions. First, we have excited the cell with the whole solar spectrum (line with circle points in Figure 3). In the second case we used a yellow filter, blocking the solar spectra below $400 \mathrm{~nm}$ (high energy photons) as shown in the line with square points in Figure 3a. The total light power in both cases was very carefully maintained at $100 \mathrm{~mW} / \mathrm{cm}^{2}$. The open circuit voltage $\left(V_{\text {oc }}\right)$ and short circuit current $\left(J_{\mathrm{sc}}\right)$ increased in the case of solar cell, which is excited with UV
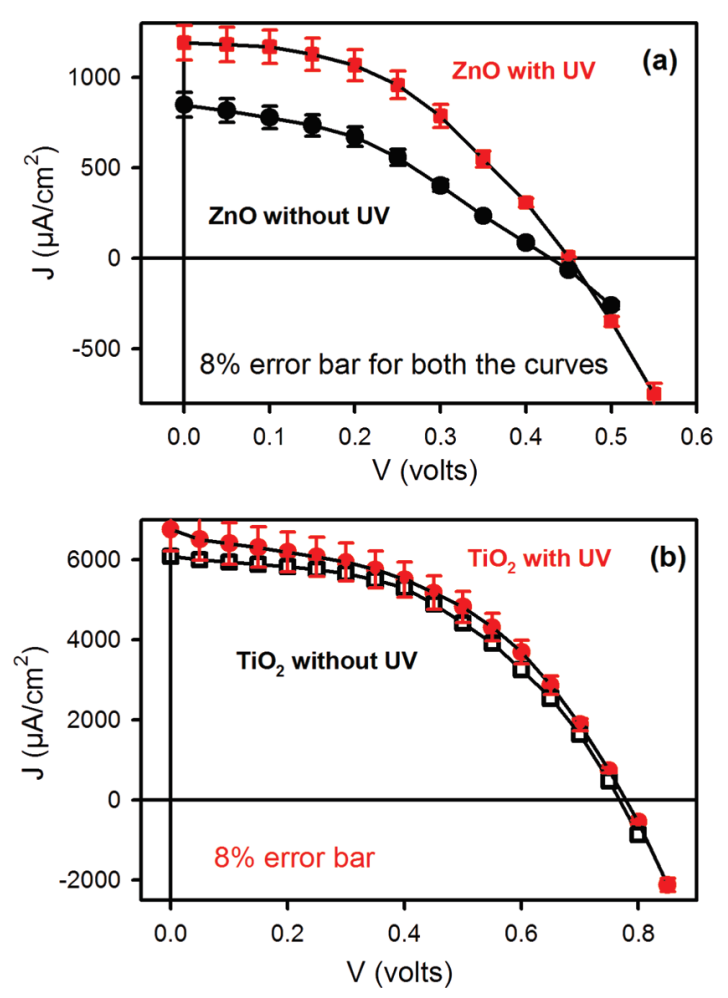

Figure 3. (a) $I-V$ curves of (a) $\mathrm{ZnO} N \mathrm{NP}$ and (b) $\mathrm{TiO}_{2} \mathrm{NP}$ based DSSC in the presence and absence of UV.

TABLE 2: Device Performance ${ }^{a}$ of the Dye-Sensitized Solar Cell with $\mathrm{ZnO}$ and $\mathrm{TiO}_{2} \mathrm{NPs}$

\begin{tabular}{lcccc}
\hline device with N719 & $V_{\mathrm{OC}}(\mathrm{V})$ & $J_{\mathrm{SC}}\left(\mu \mathrm{A} / \mathrm{cm}^{2}\right)$ & $\mathrm{FF}(\%)$ & $\eta(\%)$ \\
\hline $\mathrm{ZnO}$ NP with UV & 0.45 & 1190.00 & 44.66 & 0.24 \\
$\mathrm{ZnO}$ NP without UV & 0.42 & 668.00 & 39.39 & 0.11 \\
$\mathrm{TiO}_{2}$ NP with UV & 0.77 & 6760.00 & 46.30 & 2.41 \\
$\mathrm{TiO}_{2}$ NP without UV & 0.77 & 6086.67 & 47.15 & 2.21
\end{tabular}

${ }^{a}$ Short circuit photocurrent densities $\left(J_{\mathrm{sc}}\right)$, open-circuit voltage $\left(V_{\text {oc }}\right)$, fill factor (FF), and efficiency $(\eta)$.

radiation (without filter). Upon the use of the optical filter, a sharp decrease in current from $1190 \mu \mathrm{A} / \mathrm{cm}^{2}$ to $668 \mu \mathrm{A} / \mathrm{cm}^{2}$ was observed. In retrospect, compared to the $43.86( \pm 8) \%$ change in the short circuit current, $V_{\text {oc }}$ was reduced marginally by $6.66( \pm 4) \%$ while the fill factor $(\mathrm{FF})$ was reduced only by around $11.80( \pm 3) \%$ (Table 2). As shown in Figure 1b the SD N719 absorbs very little in the UV region (below $400 \mathrm{~nm}$ ) of the solar spectrum. Thus it is expected that the efficiency of the solar cell will not be affected much by the presence of highenergy photons due to the absence of noticeable optical absorption in the dye at lower wavelengths (below $400 \mathrm{~nm}$ ). This observation clearly demonstrates that ZnO DSSCs can harvest high-energy photons as well. High-energy photons were found to have a minimum effect on the solar cell performance of the $\mathrm{TiO}_{2}$-based solar cell with the same SD (Figure 3b). From these observations (Figure 3) and also Table 2, we can see that the efficiency of ZnO-based DSSC increases by $54.15( \pm 10) \%$ 
if the UV portion of the AM1.5 spectra is employed as well compared to a marginal $8.29( \pm 4) \%$ increase in efficiency in the case of $\mathrm{TiO}_{2}$-based DSSC. It is clear that harvesting of highenergy photons in the case of $\mathrm{TiO}_{2}$-based solar cells does not contribute appreciably to the enhancement of the device efficiency. It is worth noting that the efficiency obtained from the model DSSC with use of nanoparticles is lower compared to the maximum efficiency reported with the nanorod in order to provide higher surface area and better charge transport.

It has to be noted that N719 is a redox active organic dye. ${ }^{4}$ In this regard the PL quenching of the ZnO NPs upon adsorption of the dye may be associated with excited state electron transfer from $\mathrm{ZnO}$ to the $\mathrm{N} 719$ dye molecule. To investigate the electron transfer dynamics from the $\mathrm{ZnO}$ NPs upon excitation, we have studied the complexation of the NP with an organic molecule, benzoquinone (BQ), which is well-known as an electron acceptor $^{35}$ and efficiently accepts excited electrons from the surface of semiconductor quantum dots. ${ }^{36}$

From our studies the quenching of the PL intensity of $\mathrm{ZnO}$ NPs upon complexation with BQ is evident (data not shown). As shown in the inset of Figure 2b, a very sharp decay in fluorescence at $550 \mathrm{~nm}$ in the presence of the electron acceptor is indicative of the fast transfer of excited electrons from the conduction band of the ZnO NPs into the LUMO of BQ molecules. The $\mathrm{ZnO} / \mathrm{BQ}$ system exhibited a faster initial decay with a time period of $0.187 \mathrm{~ns}$ with a majority (76\%) of the excited electrons following this path (Table 1). The faster decay in the presence of BQ as compared to N719 at the same excitation of $375 \mathrm{~nm}$ may be indicative of energy transfer from the $\mathrm{ZnO}$ donor NPs to the N719 acceptor molecules rather than transfer of electrons. In the case of pure $\mathrm{ZnO}$ in ethanol (Figure $2 \mathrm{~b})$, the excited state lifetime $\left(k_{\mathrm{nr}}=0\right)$ of $\mathrm{ZnO}$ NPs is $20.32 \mathrm{~ns}$, which yields a radiative rate constant of $4.92 \times 10^{7}$. Upon the addition of $\mathrm{N719}$ and BQ in separate ZnO NP solution, the average lifetimes were found to reduce to 5.25 and $2.20 \mathrm{~ns}$, with rate constants of $1.90 \times 10^{8}$ and $4.55 \times 10^{8} \mathrm{M}^{-1} \mathrm{~s}^{-1}$, respectively. We have calculated the electron transfer rate, which is directly reflected in the nonradiative rate constants $\left(k_{\mathrm{nr}}\right)$. The calculated nonradiative rate constants were found to be $1.41 \times$ $10^{8}$ and $4.06 \times 10^{8} \mathrm{M}^{-1} \mathrm{~s}^{-1}$ for the $\mathrm{ZnO} / \mathrm{N} 719$ and $\mathrm{ZnO} / \mathrm{BQ}$ systems, respectively. From the above $k_{\mathrm{nr}}$ values, we can conclude that the electron transfer rate, in the case of $\mathrm{ZnO} / \mathrm{BQ}$ composite, is 3-fold higher compared to the energy transfer in the $\mathrm{ZnO} / \mathrm{N} 719$ system. In the case of electron transfer from the excited state ZnO NPs to N719, the short circuit current of the $\mathrm{ZnO}$ NP-based DSSC is expected to be reduced. However, as shown in Figure 3, the efficiency of the DSSC in the presence of UV light (i.e., when the ZnO NPs were excited) is increased. Charge conservation clearly rules out any possibility of back electron transfer from ZnO NPs to the SD N719.

\section{Conclusion}

The overall picture that is evident from our studies is represented in Scheme 1. Time-correlated single photon counting spectroscopy was carried out to understand the resonance energy transfer phenomenon in a $\mathrm{ZnO} / \mathrm{N} 719$ system, which is widely used in dye-sensitized solar cells. The semiconductor ZnO NPs was found to transfer the excited state energy to sensitizing dye N719 at the surface of the NPs. The observed energy transfer in the presence of high-energy photons leads to a significant increase in the short circuit current $(43.86( \pm 8) \%)$ in $\mathrm{ZnO}$-based DSSC leading to more than $50 \%$ increased energy conversion efficiency. The $\mathrm{TiO}_{2}$-based solar cell showed marginal increase in short circuit current and a slight efficiency improvement
SCHEME 1: A model DSSC based on the ZnO NPs, Which Contains Sufficient Defect States, Namely Oxygen Vacancy Centers near Surface Depletion Region ${ }^{a}$

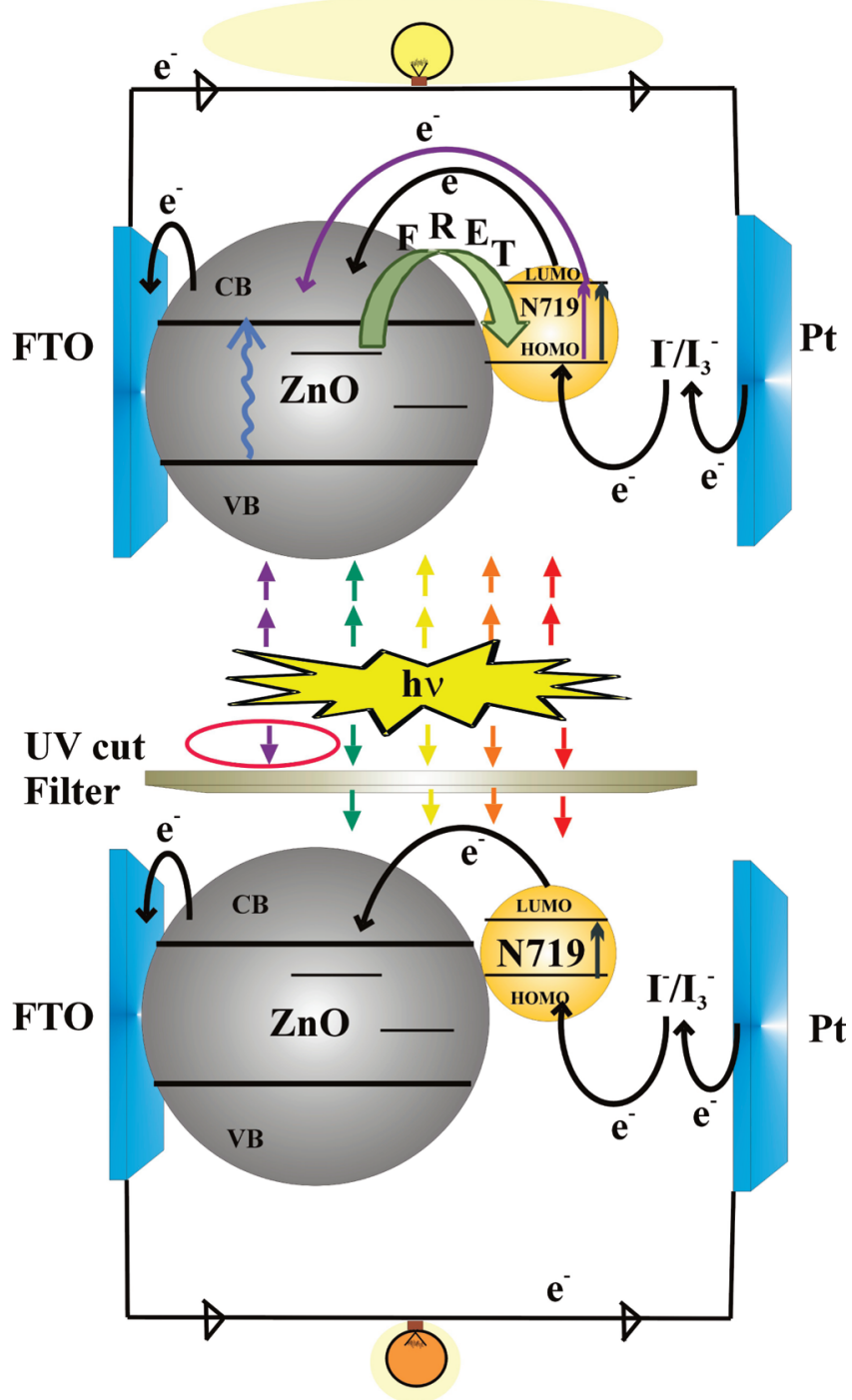

${ }^{a}$ The system consists of a fluorinated tin oxide (FTO) on which semiconductor $\mathrm{ZnO}$ nanoparticle (gray big circle) is febricated. The sensitizer dye N719 (small yellow circle) is bound to $\mathrm{ZnO}$ NP by surface adsorption. The entire structure is immersed in a liquid redox electrolyte $\left(\mathrm{I}^{-} / \mathrm{I}_{3}{ }^{-}\right)$. In regular DSSC, light is directly absorbed by the SD, exciting an electron from the HOMO to the LUMO level. In the proposed configuration that contains a semiconductor having an appreciable amount of defect states, an additional "indirect excitation" of N719 is introduced by using the sensitivity of FRET. In the FRET-based cell, the semiconductor NP is found to be excited in the presence of UV light, by which an electron is promoted from the valence band to the conduction band, followed by energy transfer from excited states of semiconductor to SD N719 via FRET. Thereafter, charge separation occurs exactly as it does in regular DSSC and an electron is injected from the excited state of the dye into the conduction band of the semiconductor electrode and the electron is thus transported to the charge collector. In the presence of a UV cut filter the FRET-based cell performs like a regular DSSC as no energy transfer from ZnO NP to SD takes place due to the absence of UV light.

$(\sim 10 \%)$. The possibility of the back electron transfer from the $\mathrm{ZnO}$ surface to the N719 has also been compared by studying the complexation of the NPs with an electron accepting organic molecule BQ. It has been found that the energy transfer process does not involve the physical migration of electron from the surface of the excited $\mathrm{ZnO}$ to the SD. Our studies may find 
relevance in the enhancement of the harvesting of the highenergy photon by semiconductors with defect centers without further addition of cosensitizer in a DSSC. The observed FRET dynamics in the light harvesting process opens the way toward the utilization of new materials which contain an appreciable number of defect states. These findings provide the importance of the various semiconductors containing inherent optical defect centers and how the defect centers can be beneficially utilized for light harvesting/sensitization.

Acknowledgment. A.M. thanks CSIR, and S.S. thanks UGC for fellowships. We thank DST for financial grants SR/SO/BB15/2007 and Indo-Thailand Project DST/INT/THAI/P06/2008 and also financial support for Unit in Nanoscience. The authors (J.D., S.B., T.B.) would like to acknowledge partial financial support from the Centre of Excellence in Nanotechnology at the Asian Institute of Technology and the National Nanotechnology Center (NANOTEC) belonging to the National Science \& Technology Development Agency (NSTDA), Thailand.

\section{References and Notes}

(1) Grätzel, M. Nature 2001, 414, 338.

(2) O’Regan, B.; Grätzel, M. Nature 1991, 353, 737.

(3) Bai, Y.; Cao, Y.; Zhang, J.; Wang, M.; Li, R.; Wang, P.; Zakeeruddin, S. M.; Grätzel, M. Nat. Mater. 2008, 7, 626.

(4) Shankar, K.; Feng, X.; Grimes, C. A. ACS Nano 2009, 3, 788.

(5) Law, M.; Greene, L. E.; Johnson, J. C.; Saykally, R.; Yang, P. Nat. Mater. 2005, 4, 455 . 4490 .

(6) Ferrere, S.; Zaban, A.; Gregg, B. A. J. Phys. Chem. B 1997, 101, 825. 3825 .

(8) Kruger, J.; Plass, R.; Gratzel, M.; Cameron, P. J.; Peter, L. M. J. Phys. Chem. B 2003, 107, 7536.

(9) Nazeeruddin, M. K.; Angelis, F. D.; Fantacci, S.; Selloni, A.; Viscardi, G.; Liska, P.; Ito, S.; Takeru, B.; Grätzel, M. J. Am. Chem. Soc. 2005, 127, 16835.

(10) Hamman, T. W.; Jensen, R. A.; Martinson, A. B. F.; Ryswyk, H. V.; Hupp, J. T. Energy Environ. Sci. 2008, 1, 66.

(11) Gratzel, M. J. Photochem. Photobiol., A 2004, 164, 3.

(12) Wang, P.; Zakeeruddin, S. M.; Moser, J. E.; Nazeeruddin, M. K.; Sekiguchi, T.; Grätzel, M. Nat. Mater. 2003, 2, 402.
(13) Yum, J. H.; Walter, P.; Huber, S.; Rentsch, D.; Geiger, T.; Nüesch, F.; Angelis, F. D.; Grätzel, M.; Nazeeruddin, M. K. J. Am. Chem. Soc. 2007, 129, 10320 .

(14) Hardin, B. E.; Hoke, E. T.; Armstrong, P. B.; Yum, J. H.; Comte, P.; Torres, T.; Frechet, J. M. J.; Nazeeruddin, M. K.; Gratzel, M.; McGehee, M. D. Nat. Photonics 2009, 3, 406.

(15) Cid, J. J.; Yum, J. H.; Jang, S. R.; Nazeeruddin, M. K.; Ferrero, E. M.; Palomares, E.; Ko, J.; Gratzel, M.; Torres, T. Angew. Chem. 2007, 46,8358 .

(16) Siegers, C.; Hohl-Ebinger, J.; Zimmermann, B.; Würfel, U.; Mülhaupt, R.; Hinsch, A.; Haag, R. Chem. Phys. Chem. 2007, 8, 1548.

(17) Keis, K.; Bauer, C.; Boschloo, G.; Hagfeldt, A.; Westermark, K.; Rensmo, H.; Siegbahn, H. J. Photochem. Photobiol. A 2002, 148, 57.

(18) Suh, D. I.; Lee, S. Y.; Kim, T. H.; Chun, J. M.; Suh, E. K.; Yang,

O. B.; Lee, S. K. Chem. Phys. Lett. 2007, 442, 348.

(19) Ranga Rao, A.; Dutta, V. Nanotechnology 2008, 19, 445712(9p).

(20) Yang, P.; Yan, H.; Mao, S.; Russo, R.; Johnson, J.; Saykally, R.;

Morris, N.; Pham, J.; He, R.; Choi, H. J. Adv. Funct. Mater. 2002, 12, 323.

(21) Xia, J. B.; Zhang, X. W. Eur. Phys. J. B 2006, 49, 415.

(22) Quintana, M.; Edvinsson, T.; Hagfeldt, A.; Boschloo, G. J. Phys. Chem. C 2007, 111, 1035.

(23) Keis, K.; Magnusson, E.; Lindström, H.; Lindquist, S. E.; Hagfeldt, A. Sol. Energy Mater. Sol. Cells 2002, 73, 51.

(24) Baruah, S.; Sinha, S. S.; Ghosh, B.; Pal, S. K.; Raychaudhuri, A. K.; Dutta, J. J. Appl. Phys. 2009, 105, 074308.

(25) Ullah, R.; Dutta, J. J. Hazard. Mater. 2008, 156, 194.

(26) Sugunan, A.; Warad, H. C.; Boman, M.; Dutta, J. J. Sol-Gel Sci. Technol. 2006, 39, 49.

(27) Baruah, S.; Dutta, J. J. Sol-Gel Sci. Technol. 2009, 50, 456.

(28) Hossain, M. K.; Ghosh, S. C.; Boontongkong, Y.; Thanachayanont, C.; Dutta, J. J. Metastable Nanocryst. Mater. 2005, 23, 27.

(29) Baruah, S.; Dutta, J. Sci. Technol. Adv. Mater. 2009, 10, 013001 (18pp).

(30) Lakowicz, J. R. Principles of Fluorescence Spectroscopy, 2nd ed.; Kluwer Academic/Plenum Publishers: New York, 1999.

(31) Braslavsky, S. E.; Fron, E.; Rodriguez, H. B.; Roman, E. S.; Scholes, G. D.; Schweitzer, G.; Valeur, B. W. J. Photochem. Photobiol. Sci. 2008, 7, 1444 .

(32) Shalish, I.; Temkin, H.; Narayanmurti, V. Phys. Rev. B 2004, 69, 245401.

(33) Ghosh, M.; Raychaudhuri, A. K. Nanotechnology 2008, 19, 445704.

(34) Narayanan, S. S.; Sinha, S. S.; Verma, P. K.; Pal, S. K. Chem.

Phys. Lett. 2008, 463, 160.

(35) Burda, C.; Green, T. C.; Link, S.; El-Sayed, M. A. J. Phys. Chem. $B$ 1999, 103, 1783.

(36) Makhal, A.; Yan, H.; Lemmens, P.; Pal, S. K. J. Phys. Chem. C 2010, 114,627 .

JP102042J 